\title{
Current trends in nanomechanical testing research
}

\author{
Benoit Merle ${ }^{1, a)}$, Verena Maier-Kiener ${ }^{2}$, Timothy J. Rupert ${ }^{3}$, George M. Pharr ${ }^{4}$ \\ ${ }^{1}$ Materials Science \& Engineering, Institute I \& Interdisciplinary Center for Nanostructured Films (IZNF), Friedrich-Alexander-Universität Erlangen- \\ Nürnberg (FAU), Erlangen, Germany \\ ${ }^{2}$ Department Materials Science, Montanuniversität Leoben, Leoben, Austria \\ ${ }^{3}$ Department of Materials Science \& Engineering, University of California, Irvine, Irvine, CA, USA \\ ${ }^{4}$ Department of Materials Science \& Engineering, Texas A\&M University, College Station, TX, USA \\ a) Address all correspondence to this author. e-mail: Benoit.merle@fau.de \\ Published online: 1 July 2021
}

\section{Introduction}

Small-scale mechanical characterization is essential for ensuring the service, performance, and lifetime of microscopic components, such as thin films and coatings, electronic sensors, and MEMS (Micro-Electro-Mechanical Systems). The first mechanical measurements on the submicrometer scale were enabled by the development of nanoindentation in the 1980s and early 1990s. From the beginning [1], Journal of Materials Research (JMR) has been the flagship journal for this field. In addition to countless contributed articles, the four previous focus issues published between 1999 and 2012 [2-5] have strongly contributed to disseminating the latest in method developments and trends in the field. This new focus issue on Advanced Nanomechanical Testing is a unique opportunity to identify and reflect upon the current research trends.

\section{Nanoindentation}

Nanoindentation has been considered the reference smallscale mechanical characterization method for almost 30 years. Its robustness has been repeatedly proven on a wide range of materials [6]. In 2002, it became the first and, to date, the only nanomechanical testing method sanctioned with an ISO norm (ISO:14,577 [7]). Still, a non-negligible number of the contributions to this focus issue are devoted to further improving its reliability [8-13], based on emerging technologies. These include more accurate imaging $[8,10,11]$ and faster data processing [9], which, for example, allow for improved characterization of the geometry of the indenter tip and the resulting contact area. In addition, this focus issue documents new method developments, which aim at further expanding the capabilities of nanoindentation beyond the measurement of hardness and Young's modulus, e.g., accessing the local creep properties [14], ductility [15], and surface free energy [16] of materials.

\section{Focused Ion Beam (FIB)-based nanomechanical testing}

Complex mechanical behaviors and associated materials parameters have become accessible through advanced nanomechanical testing techniques relying on a preliminary structuring of the samples via focused ion beam (FIB) milling. The most popular application in the focus issue is measuring the local fracture behavior of materials [17-20]. In addition to providing a desired complex microsample geometry, the FIB micromachining technique allows one to visualize the deformation field that would otherwise be concealed under the surface [21].

As evidenced by the high number of related contributions, the use of the FIB has become essential in the field of nanomechanical testing [17, 18, 20-22]. This has inevitably raised concerns about possible measurement artifacts due to ion damage incurred during the specimen preparation [20, 23-25]. While detrimental effects can be ruled out for some applications [20], in other cases, workarounds are actively being developed to minimize [24] or to completely avoid [23] exposure of the samples to highly energetic ion beams.

\section{Big data}

A significant trend pervading this focus issue is the strongly increasing use of nanoindentation as a high-throughput method for producing large amounts of data [26-31], which are then analyzed with emerging data science algorithms. The former is made possible through recent technical developments by hardware manufacturers which aim at performing a single indentation within no more than $1 \mathrm{~s}$ (KLA: Express Test and NanoBlitz, Bruker: XPM, Alemnis: UHSR, and others). This allows for the measurement of large mappings of up to 100,000 indents within 1-2 days. Such large datasets are then statistically analyzed, e.g., by machine learning algorithms [26]. The aim of such studies 
is generally to access the intrinsic mechanical properties of the different components of modern alloys and composite materials.

\section{Correlative investigations}

Because a statistical analysis does not always yield an unequivocal identification of the material phases, there is a growing trend of performing correlative analyses, which combine nanoindentation mapping with imaging of the sample surface using one or more analytical techniques [27-29, 32, 33]. Alternatively, numerical simulations can be used to inform the experiments and data analysis $[32,34]$. The most widely used imaging techniques are backscattered electron diffraction (EBSD) for mapping the crystallographic orientation and energy dispersive $\mathrm{X}$-ray spectroscopy (EDX) for local chemical characterization [27-29, 32, 33]. This focus issue also witnesses the use of other emerging techniques, such as atom probe tomography (APT), which provides chemical composition mapping with strongly enhanced spatial resolution $[29,33]$. Regardless of the nanomechanical testing method, post-mortem analyses appear to greatly benefit from the increasing availability of image processing algorithms, such as digital image correlation $[11,25,35,36]$.

\section{In-situ investigations}

In-situ experiments in a microscope offer an attractive alternative to combinatorial approaches, since they allow one to unequivocally correlate a given mechanical behavior to a given deformation mechanism. Their development has been a dominant trend in nanomechanical research throughout the past decade, with their application seemingly only being limited by the high costs associated with the techniques. This trend is continued in the focus issue, which features applications both inside scanning electron microscopes (SEM) $[17,18,21,22]$ and transmission electron microscopes (TEM) $[23,24]$.

\section{Extreme testing environments}

Combining high spatial resolution with extreme environments is arguably the greatest experimental challenge currently faced by the nanomechanical testing community. Owing to intense research and development activities during the past decade, nanoindentation has been successfully expanded to applications at high temperatures. This has proven highly beneficial for investigating not only the deformation behavior of high-temperature materials [37, 38], but also the fundamental effects of heat treatments [31]. In addition, the focus issue features a study conducted at cryogenic temperatures [39], which underscores that progress is also being made in this direction. Environmentally controlled experimental setups also allow investigating the influence of humidity [19] on mechanical properties. The frontier of extreme testing currently appears to lie with very high strain rates [40] and very high cycle fatigue [41] applications, with both requiring significant improvements to hardware in order to deal with the acquisition of mass data within a short time, as well as with their subsequent evaluation.

\section{Conclusions}

This focus issue on Advanced Nanomechanical Testing documents how actively developments are taking place in the field of nanomechanical testing research. Most of the current developments aim at deriving benefits from the latest advancements in data acquisition, data processing, as well as the new environmental capabilities of nanoindentation hardware. The overarching goals are to improve the statistical significance of the results and to reproduce the extreme environments that are responsible for the failure of many systems.

In summary, this focus issue presents a diverse perspective of the challenges and opportunities offered at the frontiers of nanomechanical testing, which we hope demonstrates to the reader the exciting future of this topic.

\section{Acknowledgments}

We would like to acknowledge the dedication of the many anonymous reviewers who have enforced the high scientific standards of this focus issue.

\section{References}

1. M.F. Doerner, W.D. Nix, A method for interpreting the data from depth-sensing indentation instruments. J. Mater. Res. 1, 601-609 (1986)

2. A.B. Mann, R.C. Cammarata, M.A. Nastasi, Focus issue: nanoindentation. J. Mater. Res. 14, 2195 (1999)

3. Cheng, Y.-T., Page, T., Pharr, G. M., Swain, M. V. \& Wahl, K. J. Focus Issue: Fundamentals and Applications of Instrumented Indentation in Multidisciplinary Research. J. Mater. Res. 19, (2004).

4. G.M. Pharr et al., Focus issue: indentation methods in advanced materials research. J. Mater. Res. 24, 3628 (2009)

5. D. Kwon, M.M. Chaudhri, Y.-T. Cheng, O. Kraft, Instrumented indentation. J. Mater. Res. 27, 1-388 (2012)

6. W.C. Oliver, G.M. Pharr, Measurement of hardness and elastic modulus by instrumented indentation: advances in understanding and refinements to methodology. J. Mater. Res. 19, 3-20 (2004)

7. Metallic materials-Instrumented indentation test for hardness and materials parameters-Part 1: Test method. Int. Stand. ISO 14577-1 (2002) 
8. C. Saringer, M. Tkadletz, M. Kratzer, M.J. Cordill, Direct determination of the area function for nanoindentation experiments. J. Mater. Res. (2021). https://doi.org/10.1557/ s43578-021-00113-9

9. P. Sudharshan Phani, W.C. Oliver, G.M. Pharr, Measurement of hardness and elastic modulus by load and depth sensing indentation: Improvements to the technique based on continuous stiffness measurement. J. Mater. Res. (2021). https://doi.org/10.1557/ s43578-021-00131-7

10. J.E. Jakes, D. Stauffer, Contact area correction for surface tilt in pyramidal nanoindentation. J. Mater. Res. (2021). https://doi.org/ 10.1557/s43578-021-00119-3

11. S. Brinckmann, R. Schwaiger, Towards enhanced nanoindentation by image recognition. J. Mater. Res. (2021). https://doi.org/ 10.1557/s43578-021-00173-x

12. J. Wang, T. Volz, S.M. Weygand, R. Schwaiger, The indentation size effect of single-crystalline tungsten revisited. J. Mater. Res. (2021). https://doi.org/10.1557/s43578-021-00221-6

13. S. Fulco, S. Wolf, J.E. Jakes, Z. Fakhraai, K.T. Turner, Effect of surface detection error due to elastic-plastic deformation on nanoindentation measurements of elastic modulus and hardness. J. Mater. Res. (2021). https://doi.org/10.1557/ s43578-021-00223-4

14. Z.Y. Liang, S.S. Xiang, G.M. Pharr, Effects of crystal orientation on the indentation creep of $\beta$-tin. J. Mater. Res. (2021). https:// doi.org/10.1557/s43578-021-00165-x

15. C. Minnert, H. ur Rehman, K. Durst, Thermally activated dislocation mechanism in Mo studied by indentation, compression and impact testing. J. Mater. Res. (2021). https://doi.org/10.1557/ s43578-021-00126-4

16. E.M. Rossi et al., A novel nanoindentation protocol to characterize surface free energy of superhydrophobic nanopatterned materials. J. Mater. Res. (2021). https://doi.org/10.1557/ s43578-021-00127-3

17. C. Tian, C. Kirchlechner, The fracture toughness of martensite islands in dual-phase DP800 steel. J. Mater. Res. (2021). https:// doi.org/10.1557/s43578-021-00150-4

18. M. Burtscher et al., In situ fracture observations of distinct interface types within a fully lamellar intermetallic TiAl alloy. J. Mater. Res. (2021). https://doi.org/10.1557/s43578-020-00088-Z

19. S. Jaddi, J.-P. Raskin, T. Pardoen, On-chip environmentally assisted cracking in thin freestanding $\mathrm{SiO} 2$ films. J. Mater. Res. (2021). https://doi.org/10.1557/s43578-021-00189-3

20. E.I. Preiß et al., Applicability of focused Ion beam (FIB) milling with gallium, neon, and xenon to the fracture toughness characterization of gold thin films. J. Mater. Res. (2021). https://doi.org/ 10.1557/s43578-020-00045-w

21. R.M.G. De Meyere et al., A novel trench fibre push-out method to evaluate interfacial failure in long fibre composites. J. Mater. Res. (2021). https://doi.org/10.1557/s43578-021-00153-1
22. I. Bikmukhametov, T.R. Koenig, G.J. Tucker, G.B. Thompson, A rapid preparation method for in situ nanomechanical TEM tensile specimens. J. Mater. Res. (2021). https://doi.org/10.1557/ s43578-021-00167-9

23. N.R. Velez et al., Nanomechanical testing of freestanding polymer films: in situ tensile testing and Tg measurement. J. Mater. Res. (2021). https://doi.org/10.1557/s43578-021-00163-z

24. Y. Yang et al., Evaluating the effects of pillar shape and gallium ion beam damage on the mechanical properties of single crystal aluminum nanopillars. J. Mater. Res. (2021). https://doi.org/10. 1557/s43578-021-00125-5

25. P. Hosemann et al., Quantifying residual stress in Heliumimplanted surfaces and its implication for blistering. J. Mater. Res. (2021). https://doi.org/10.1557/s43578-021-00108-6

26. H. Besharatloo, J.M. Wheeler, Influence of indentation size and spacing on statistical phase analysis via high-speed nanoindentation mapping of metal alloys. J. Mater. Res. (2021). https://doi. org/10.1557/s43578-021-00214-5

27. Z. Liu, J. Zhang, B. He, Y. Zou, High-speed nanoindentation mapping of a near-alpha titanium alloy made by additive manufacturing. J. Mater. Res. (2021). https://doi.org/10.1557/ s43578-021-00204-7

28. C.M. Magazzeni et al., Nanoindentation in multi-modal map combinations: a correlative approach to local mechanical property assessment. J. Mater. Res. (2021). https://doi.org/10.1557/ s43578-020-00035-y

29. A. Orozco-Caballero, C. Gutierrez, B. Gan, J.M. Molina-Aldareguia, High-throughput nanoindentation mapping of cast IN718 nickel-based superalloys: influence of the $\mathrm{Nb}$ concentration. J. Mater. Res. (2021). https://doi.org/10.1557/s43578-021-00133-5

30. K.M. Schmalbach et al., Nanomechanical mapping and strain rate sensitivity of microcrystalline cellulose. J. Mater. Res. (2021). https://doi.org/10.1557/s43578-021-00138-0

31. G. Tiphéne et al., High-Temperature Scanning Indentation: A new method to investigate in situ metallurgical evolution along temperature ramps. J. Mater. Res. (2021). https://doi.org/10. 1557/s43578-021-00107-7

32. T. Keil et al., Solid solution hardening in CrMnFeCoNibased high entropy alloy systems studied by a combinatorial approach. J. Mater. Res. (2021). https://doi.org/10.1557/ s43578-021-00205-6

33. H.M. Gardner et al., Quantifying the effect of oxygen on micromechanical properties of a near-alpha titanium alloy. J. Mater. Res. (2021). https://doi.org/10.1557/s43578-020-00006-3

34. W. Yang, D. Lacroix, L.P. Tan, J. Chen, Revealing the nanoindentation response of a single cell using a 3D structural finite element model. J. Mater. Res. (2021). https://doi.org/10.1557/ s43578-020-00004-5

35. M. Liu, I. McCue, M.J. Demkowicz, Quantifying surface deformation around micrometer-scale indents by digital image 
correlation. J. Mater. Res. (2021). https://doi.org/10.1557/ s43578-020-00019-y

36. M. Alfreider, M. Meindlhumer, V. Maier-Kiener, A. Hohenwarter, D. Kiener, Extracting information from noisy data: strain mapping during dynamic in situ SEM experiments. J. Mater. Res. (2021). https://doi.org/10.1557/s43578-020-00041-0

37. J. Kappacher, O. Renk, D. Kiener, H. Clemens, V. Maier-Kiener, Controlling the high temperature deformation behavior and thermal stability of ultra-fine-grained $\mathrm{W}$ by re alloying. J. Mater. Res. (2021). https://doi.org/10.1557/s43578-020-00026-Z

38. C.H. Bumgardner, J.D. Baker, A.J. Ritchey, X. Li, Probing the local creep mechanisms of $\mathrm{SiC} / \mathrm{SiC}$ ceramic matrix composites with high-temperature nanoindentation. J. Mater. Res. (2021). https://doi.org/10.1557/s43578-021-00128-2
39. G. Song, N.K. Aragon, I. Ryu, S. Lee, Low-temperature failure mechanism of [001] niobium micropillars under uniaxial tension. J. Mater. Res. (2021). https://doi.org/10.1557/ s43578-020-00069-2

40. R. Ramachandramoorthy et al., High strain rate in situ micropillar compression of a Zr-based metallic glass. J. Mater. Res. (2021). https://doi.org/10.1557/s43578-021-00187-5

41. A. Barrios, E. Kakandar, G. Castelluccio, O.N. Pierron, Comparison of the low and high/very high cycle fatigue behaviors in Ni microbeams under bending. J. Mater. Res. (2021). https://doi. org/10.1557/s43578-020-00097-y 